\title{
ATIVIDADE DE FORRAGEAMENTO DE HETEROTERMES TENUIS (HAGEN) (ISOPTERA: RHINOTERMITIDAE) EM CANA-DE-AÇÚCAR UTILIZANDO A ARMADILHA TERMITRAP®
}

\author{
J.E.M. Almeida ${ }^{1}$; S.B. Alves† \\ ${ }^{1}$ Instituto Biológico, Centro Experimental Central, CP 70, CEP 13001-970, Campinas, SP, Brasil. E-mail: \\ jemalmeida@biologico.sp.gov.br
}

\section{RESUMO}

\begin{abstract}
O objetivo deste trabalho foi estudar a atividade de forrageamento de Heterotermes tenuis na região de Piracicaba, SP, Brasil, utilizando a armadilha Termitrap ${ }^{\circledR}$ na cultura da cana-de-açúcar. Os experimentos foram instalados em áreas diferentes na fazenda Retiro. $\mathrm{Na}$ área 1 foram utilizados $2.500 \mathrm{~m}^{2}$, onde foram instaladas 40 armadilhas Termitrap ${ }^{\circledR}$ a cada 10 metros, num caminhamento em forma de $X$, cobrindo toda a área. Na área 2, com a mesma dimensão, foram instaladas 30 armadilhas, como na área 1, mas num caminhamento em forma de Z. As armadilhas Termitrap ${ }^{\circledR}$ foram elaboradas de papelão corrugado, enrolando-se um pedaço de $15 \times 150 \mathrm{~cm}$ formando um cilindro com $15 \mathrm{~cm}$ de altura e oito $\mathrm{cm}$ de diâmetro. As avaliações foram realizadas, semanalmente, durante 24 meses (1992-1993) na área 1 e durante 32 meses na área 2 (1993-1995). $\mathrm{Na}$ área 1 verificou-se uma alta correlação entre o aumento da população de operários e soldados coletados nas armadilhas com o aumento da precipitação pluviométrica e da temperatura e na área 2 houve correlação positiva apenas com a temperatura. A reforma total da área do canavial, com ações mecânicas de aração e gradagem, influenciou no forrageamento e galerias de $H$. tenuis, porém não no controle da praga.
\end{abstract}

PALAVRAS-CHAVE: Ecologia, cupim, forrageamento.

\section{ABSTRACT}

THE FORAGING ACTIVITY OF HETEROTERMES TENUIS (HAGEN) (ISOPTERA: RHINOTERMITIDAE) IN SUGARCANE USING THE TERMITRAP ${ }^{\circledR}$ TRAP. The objective of this research was to study the foraging activity of the termite Heterotermes tenuis in the region of Piracicaba, State of São Paulo, Brazil, using the Termitrap ${ }^{\circledR}$ trap. The experiments were installed in different areas of Retiro Farm. At area 1, 40 traps were installed at 10-meter intervals, in X distribution, throughout all the area, which measured $2,500 \mathrm{~m}^{2}$. At area 2, with the same dimension, 30 traps were installed, the same as in area 1, but in $\mathrm{Z}$ distribution. The traps were made of corrugated cardboard with a height of $15 \mathrm{~cm}$ and diameter of $8 \mathrm{~cm}$. The evaluations were made every week for 24 months (1992-1993) in area 1 and for 32 months (1993-1995) in area 2. In area 1 a correlation was verified between the population of termites and rainfall and temperature. In area 2 the termite's population was correlated only with temperature. The total reform of the sugarcane crop with mechanical techniques influenced in the foraging and galleries of $H$. tenuis, but did not affect the pest control.

KEY WORDS: Ecology, termite, foraging.

\section{INTRODUÇÃO}

O cupim Heterotermes tenuis é considerado uma praga importante para a cultura da cana-de-açúcar, devido a sua vasta distribuição em canaviais dos estados de São Paulo, Mato Grosso do Sul, Minas Gerais, Goiás e Paraná, e por ser a espécie mais relacionada a danos em cana (ALMEIDA et al., 1989; ARRIGONI et al., 1989).

Segundo NovARETti (1985), os ataques na cana-deaçúcar eos danos podem ser de três tipos: 1-logo após o plantio, no colmo-semente e nas primeiras raízes, causando falhas na germinação e diminuição do vigor das plantas; 2-na maturação, quando os cupins penetram

${ }^{2}$ Universidade de São Paulo, Escola Superior de Agricultura "Luiz de Queiroz", Departamento de Entomologia, Piracicaba, SP, Brasil.

tIn memorian. 
noscolmos, provocandosecamentoesua morte; e3-nas socas, quandoestãovulneráveis, atacandoorizoma. Os danos causados por cupins chegam à redução de 10 ton/ha/ano, daí a necessidade de controle.

Devidoaohábitosubterrâneo, com forrageamento pelo solo e ninho difuso, pouco se sabe sobre o comportamento da população de $H$. tenuis durante o ano e após tratos culturais da cultura da cana-de-açúcar.

Alguns pesquisadores já desenvolveram armadilhas/iscas atrativas para se estudar a biologia, ecologia e o hábito de forrageamento de cupins subterrâneos. Por exemplo, KING;SPINK (1975) estudaramabiologia de Coptotermes formosanus utilizando estacas de pinho de 2,5 x 2,5 x $29 \mathrm{~cm}$ enterradas no solo para a coleta de alados para a formação de casais e a partir de um casal, observaram que a oviposição ocorreu entre 13 a 109 dias em uma colônia e não houve oviposição durante os meses de inverno no primeiro ano.

FRENCH et al. (1981) utilizaram, como iscas, rolos de papel higiênico e blocos de madeira de Pinus sp. em uma floresta de Callitris, Casuarina e Eucalipto na Austrália. Essas iscas permaneceram no campo por 13 meses. Observaram que a espécie Heterotermes ferox foi a mais comum, infestando a maioria das armadilhas em todos os locais. Foram coletadas outras espécies, como Armitermes xylophagus, Microtermes sp. e Schedorhinotermes reticulatus.

THOMPSON (1985) realizou um monitoramento de solo na área de um condomínio na Flórida com estacas de pinho amarelo de 4,0 × 2,0 x $60 \mathrm{~cm}$ enterradas 30 cm no soloe verificou a infestação por C. formosanus, Reticulitermes flavipes e Incisitermes.

JONEs et al. (1987) utilizaram rolos de papel higiênico para estudar o forrageamento de H. aureus, colocados de 6 em 6 metros no solo. Verificaram que o ataque pelos cupins foi generalizado, demonstrando que a distância não constitui um fator fundamental para o forrageamento. Observaram que a variação sazonal influenciou no forrageamento.

O estudo da bioecologia de cupins com iscas atrativas permite estimar a área de forrageamento da colônia de cupim, bem como o tamanho desta. GRACE et al. (1989) utilizaram iscas de Pinus de 1,5 x 1,5 x 15 $\mathrm{cm}$ para capturar $R$. flavipes numa área infestada na região metropolitana de Toronto, Canadá. Esses autores marcaram uma grande quantidade deindivíduos com Fat Red 7 B, em papelão corrugado, de dois sítios de forrageamento e os soltaram nos mesmos locais da captura. Com a recaptura, verificaram que as galerias se estendiam a $79 \mathrm{~m}$ do ponto de soltura, estimando a área de forrageamento de $1.091 \mathrm{~m}^{2}$ para uma das colônias. As duas colônias estudadas possuíam cerca de 2,1 e 3,2 milhões de indivíduos.

HaAgsma; Rust (1995) estimaram o tamanho da colônia, a atividade de forrageamento, a fenologia e os parâmetros fisiológicos do cupim $R$. hespereus, numa área urbana enatural em Riverside, California. Utilizaram uma isca de papelão corrugado para a captura de insetos. Oforrageamento ocorreu demaioa outubroem condições delaboratórioeem ambientenaturalocorreu de novembro a janeiro na estação seca. Na área urbana a colônia foi estimada em 830.531 indivíduos comparada com uma área natural, com 103.758 indivíduos.

Com a finalidade de desenvolver uma armadilha eficaz na atração de $H$. tenuis e para ser usada no controle desta praga em cana no Brasil, AlmEIDA; Alves (1995) testaram vários materiais em experimentos de múltipla escolha em laboratório e campo de cana-deaçúcar em Piracicaba, SP. Verificaram que a armadilha elaborada com papelão corrugado (Termitrap $\left.{ }^{\circledR}\right)$ foi a mais atrativa para $H$.tenuis. Além desta espécie, verificaram que os cupins dos gêneros Cornitermes, Procornitermes, Coptotermes, Neocapritermes e Nasutitermes foram atraídos. Essa armadilha foi desenvolvida com o intuito de monitorar $H$. tenuis no campo e para estudos de controle com produtos microbianos associados ou não com inseticidas químicos.

O objetivo deste trabalho foi estudar o forrageamento do cupim $H$. tenuis na região de Piracicaba, SP, utilizando a armadilha Termitrap ${ }^{\circledR}$.

\section{MATERIALEMÉTODOS}

Os experimentos foram instalados em áreas diferentes na fazenda Retiro, região de Piracicaba, SP (Latitude $22^{\circ} 42^{\prime}$, Longitude $47^{\circ} 38^{\prime}$ e altitude $580 \mathrm{~m}$ ). Na área 1, com cana de terceiro corte, foram utilizados $2.500 \mathrm{~m}^{2}$, onde foram instaladas 40 armadilhas Termitrap ${ }^{\circledR}$ de 10 em 10 m, num caminhamento em forma de $X$, cobrindo toda a área.

Na área 2, com a mesma dimensão e características culturais da área 1, foram instaladas 30 armadilhas, de 10 em 10 m num caminhamento em forma de Z. As armadilhas Termitrap foram confeccionadas empapelão corrugado, enrolando-se um pedaço de 15 x $150 \mathrm{~cm}$, ficando um cilindro com $15 \mathrm{~cm}$ de altura e $8 \mathrm{~cm}$ de diâmetro.

As avaliações foram realizadas semanalmente, durante 24 meses (1992-1993) na área 1 e durante 32 meses na área 2 (1993-1995). As armadilhas com $H$. tenuis eram retiradas e levadas para a contagem dos indivíduos em laboratório, sendo substituídas no momento da coleta.

Na área 2, nos meses de setembro e outubro de 1994, foi realizada a reforma do canavial, com as seguintes prática culturais: aração, gradagem pesada, gessagem, torta de filtro, gradagem pesada eleve novamente, sulcamento e plantio, ocorrendo no dia 20 de outubro de 1994.

Os dados foram analisados correlacionando-se graficamente o número de cupins coletados com a 
temperatura, precipitação pluviométrica e os tratos culturais normais da cana-de-açúcar, tais como colheita manual, reforma com implementos de aração, gradagem esulcagem. Estudou-seoíndice de agregação das colônias, bem como a atividade da população de H. tenuis antes e após os tratos culturais do canavial. Realizaram-se também observações de ataques de predadores e parasitoides.

Osdadosmeteorológicosforam registrados pelaestação de bioclimatologia da ESALQ/USP, Piracicaba, SP.

\section{RESULTADOSEDISCUSSÃO}

Naárea 1, verificou-se nos quatro primeiros meses de 1992 uma população de cupins que variou de 60 a 250 indivíduos por isca, quando ocorreu uma temperatura média de $23^{\circ} \mathrm{C}$ e precipitação pluviométrica média de 3,96 mm/alt. A cana-de-açúcar nesta área estava na terceira safra (corte), portanto, o solo não foi revolvido durante três anos consecutivos, apenas para a capina de rotina (Fig. 1).
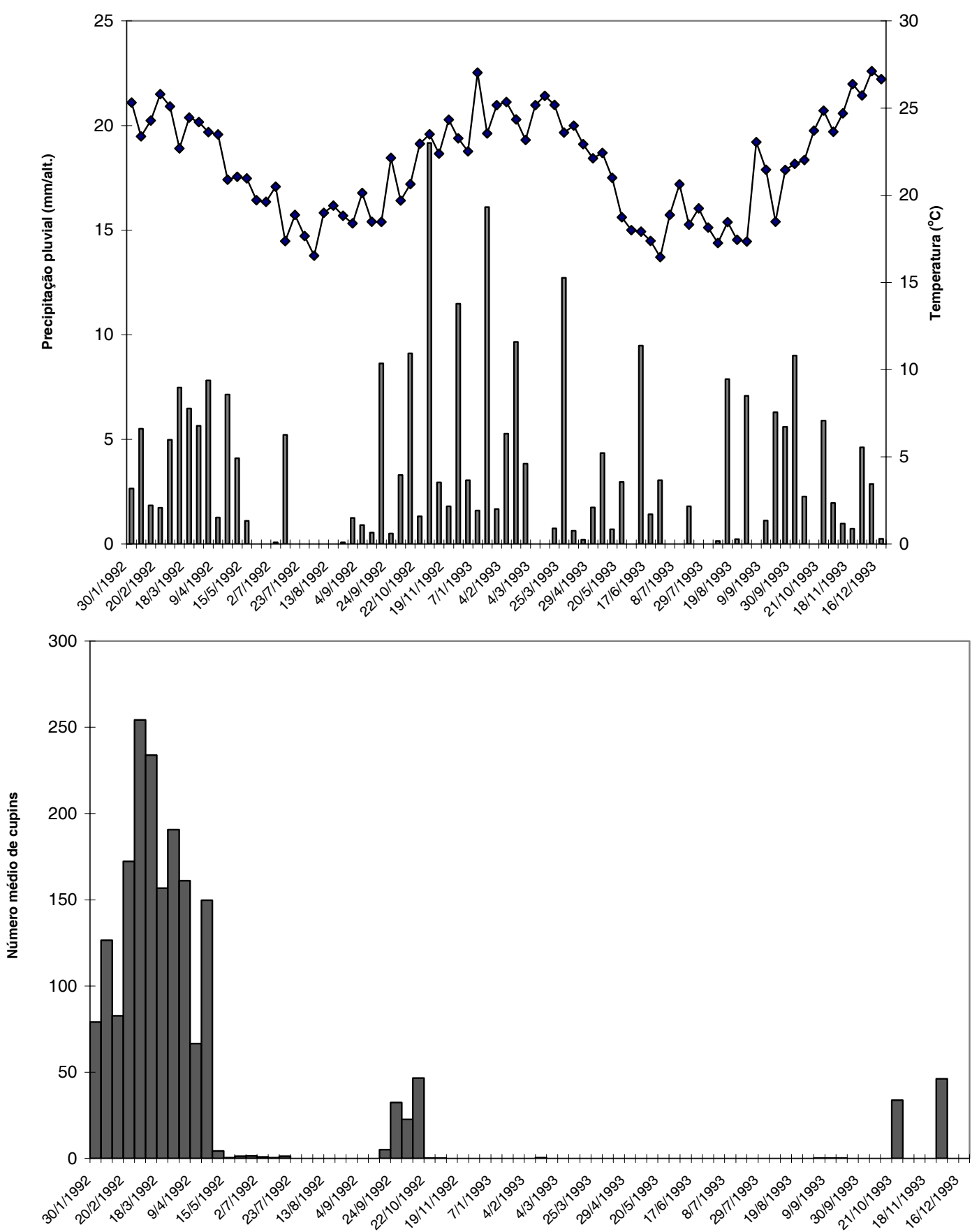

Fig. 1 - Número médio de cupins Heterotermes tenuis por armadilha na área 1 de cana-de-açúcar com a armadilha Termitrap ${ }^{\circledR}$, temperatura e precipitação pluviométrica durante as coletas de cupins no período (1992-1993) (Piracicaba, $\mathrm{SP})$. 
Nomês demaio de 1992, foi realizado um "cultivo mínimo" para um novo plantio, utilizando-se herbicida Glifosate para a destruição da soca e novo plantio da cultura. Neste período, verificou-se a diminuição da população com coletas de até 250 indivíduos por armadilha em média, para próximo de zero, decorrentes dos tratos culturais. Durante os meses de junho, julho e agosto, a média do número de cupins coletados decresceu mais ainda, chegando a zero, decorrente da diminuição da temperatura e da precipitação pluvial nos meses de inverno. Nos meses de setembro e outubro, com as chuvas de primavera, verificou-se a ocorrência de cupins nas armadilhas, mascom a sequência de uma reforma total docanavial e do plantio da nova cana (sulcagem e plantio), o número reduziu a zero novamente, permanecendo assim até fevereiro de 1993, quando foram coletados alguns indivíduos. No entanto, nos meses subsequentes, com a queda da temperatura e precipitação, o número de $H$. tenuis coletados continuou baixo, próximo de 50 indivíduos por armadilha em média. Nesta área, a população voltou a subir somente em outubro e dezembro de 1993, com o início das chuvas de verão (Fig. 1).
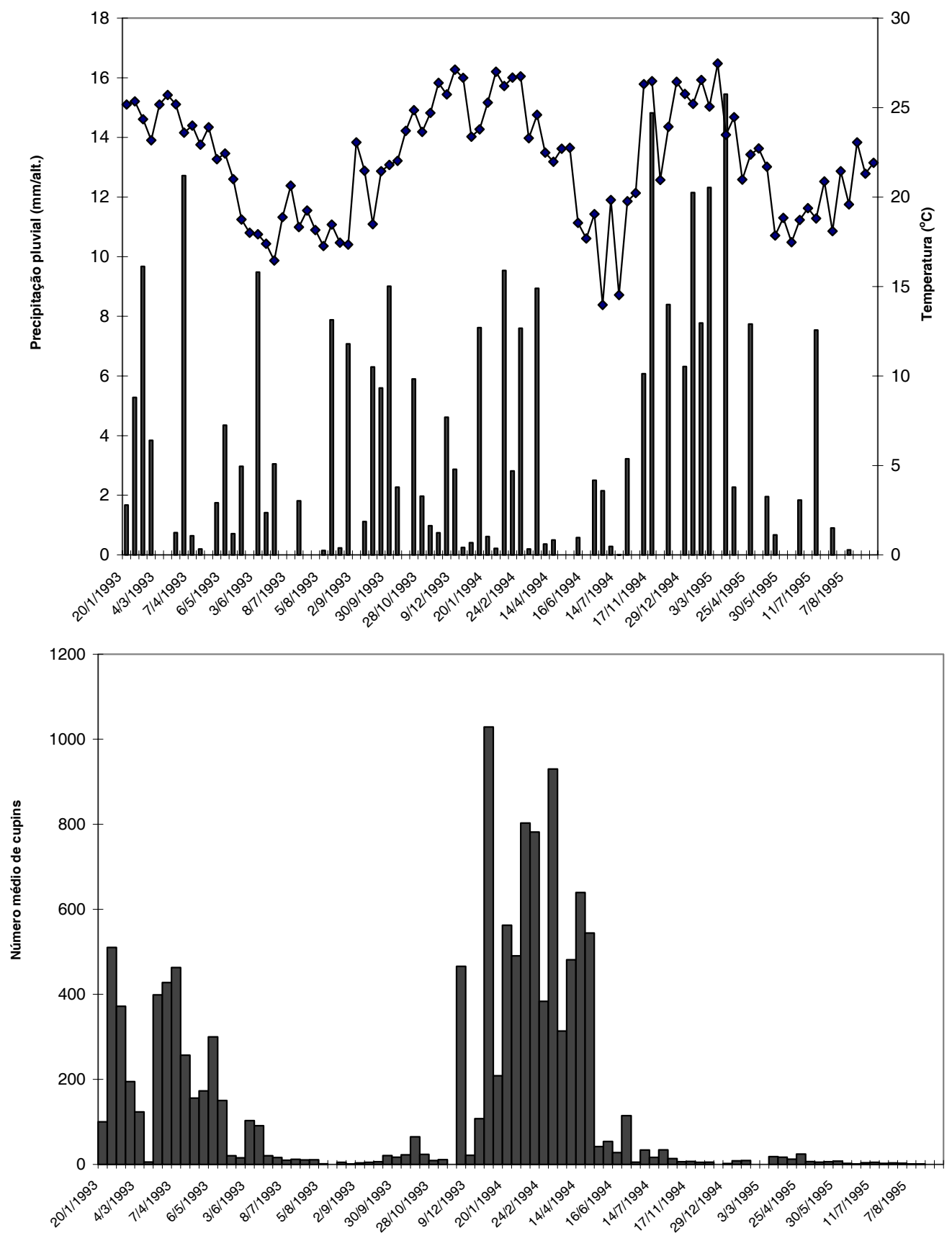

Fig. 2 - Número médio de cupins Heterotermes tenuis na área 2 de cana-de-açúcar com a armadilha Termitrap ${ }^{\circledR}$, temperatura e precipitação pluviométrica durante a coleta de no período (1993-1995) (Piracicaba, SP). 
Tabela 1 - Número médio de Heterotermes tenuis em armadilhas Termitrap ${ }^{\circledR}$ na região de Piracicaba, SP, no período de janeiro a maio dos anos de 1993, 1994 e 1995 total de duas áreas de monitoramento.

\begin{tabular}{cc}
\hline Ano de coleta & Média populacional $^{1}$ \\
\hline 1994 & $448,61 \mathrm{a}$ \\
1993 & $140,91 \mathrm{~b}$ \\
1995 & $6,52 \quad \mathrm{c}$ \\
\hline
\end{tabular}

${ }^{1}$ Médias seguidas de mesma letra não diferem entre si pelo teste de Tukey a $5 \%$.

$\mathrm{CV}=24,49 \%$

Verificou-se quehouveuma correlação donúmero de cupins coletados com a temperatura e a precipitação $\left(r^{2}=97,71 \%\right.$ e $r^{2}=94,78 \%$, respectivamente), ou seja, conforme a temperatura e a precipitação pluviométrica aumentaram, elevou-se também onúmero de cupins coletados nas armadilhas.

$\mathrm{Na}$ área 2, constatou-se maior população de $H$. tenuis, já que a média do número de cupins foi muito superior à encontrada na área 1 (Tabela 1 ). A cana-deaçúcar da área 2 estava na terceira safra (corte) em janeiro de 1993, sendo realizado o quarto corte em 1994 e a reforma do canavial em setembro e outubro de 1994. As duas áreas possuíam as mesmas características.

As avaliações da área 2 tiveram início em janeiro de 1993, quando foi observada uma média de 100 indivíduos por armadilha. Com uma temperatura média de $24^{\circ} \mathrm{C}$ e alta precipitação, a média de cupins coletados chegou a 359 cupins por armadilha no mês de fevereiro e até maio a população manteve-se alta, quando foram coletados em média 121 cupins (Fig. 2).

Nos meses de junho a setembro de 1993, notou-se uma brusca diminuição da média populacional acompanhada pela diminuição da precipitação e da temperatura. Verificou-se que, com as chuvas em outubro, a população média de cupins voltou a subir, não sendo influenciado pela colheita da cana, realizado em novembro de 1993. No verão de 1994, o número médio de cupins foi muito alto, chegando à média de 622 indivíduos por armadilha no mês de março e mantendo-se alto até junho (Fig. 2).

Novamente, observou-sea diminuição donúmero médio de cupins nas armadilhas nos meses de junho, julho e agosto, numa época de baixa precipitação, principalmente no mês deagosto, quando esteíndice foi zero (Fig. 2).

Com a reforma do canavial, ocorreu a desestruturação das colônias de cupins, com a diminuição do número decupins nas iscas, chegando a zerono mês de fevereirode1995, apesarda média detemperaturaserde $24,9^{\circ} \mathrm{C}$ e a precipitação pluviométrica de $14,84 \mathrm{~mm} /$ alt.
Verificou-se que a população voltou a subir no mês de abril de 1995, mas logo com a chegada do inverno, a média do número de indivíduos coletados reduziu novamente chegando a apenas 11 indivíduos por armadilha no mês de agosto (Fig. 2).

Apesar do número coletado ter sido maior em abril de 1995, foi possível observar que houve uma diminuição da população detectada pela mesma coleta com a reforma total do canavial, já que as práticas de aração e gradagem, bem como a gessagem e a aplicação de torta de filtro, desestruturaram o solo, afetando os ninhos de $H$. tenuis, diminuindo a atividade e coleta dos indivíduos da colônia. Essas reformas do canavial podem implicar também em diminuição temporária da oferta de alimento, maiores flutuações de temperatura e umidade do solo, entre outras mudanças que podem afetar as populações de cupins.

Observou-se que nos anos de 1993 e 1994, no período de janeiro a maio, o número de cupins coletados foi maior do que nos demais meses do ano, sendo que, em 1994, quarto ano do canavial, a população de $H$. tenuis coletada foi superior aos anos de 93 e 95. Analisando-se os dados de coleta de cupim no mesmo período no ano de 1995, verificou-se que o número de cupins coletados nas armadilhas foi muito menor que nos anos de 1993 e 1994 (Tabela 1). Esses dados demonstram que após a reforma do canavial a população de $H$. tenuis próxima à superfície do solo diminuiu muito, não sendo, portanto, necessária a aplicação de inseticidas na ocasião do plantio.

A correlação entre o número de cupins coletados ea temperatura foi positiva $\left(r^{2}=99,93 \%\right)$, mas ocorreu uma baixa correlação do número de cupins com a precipitação pluvial $\left(r^{2}=23,89 \%\right)$.

Segundo o que foi observado por AHMAD et al. (1982), a umidade relativa do ar exerce uma grande influência sobre os cupins da espécie $H$. indicola demonstrando que nos meses em que a temperatura é mais elevada, com média de precipitação maior, a atividade desses insetos também é mais elevada.

Campos (2004) também verificou que o forrageamento de $H$. tenuis em cana-de-açúcar em Araras, SP, foi interrompido drasticamente quandoo canavial foi reformado, havendo influência direta das ações mecânicas de aração e gradagem do solo sobre galerias, porém, após um período de descanso, os indivíduos sobreviventes voltaram a forragear e atacar as raízes da cana a partir do mês de outubro.

Além de $H$. tenuis, durante o levantamento foi possível coletar nas armadilhas as seguintes espécies de cupins: Cornitermes cumulans, Procornitermes sp., Nasutitermes sp., Syntermes sp., Neocapritermes sp., Ruptitermes sp. e Anoplotermes sp. Coletaram-se também seis casais de alados de $H$. tenuis no mês de novembro de 1994, na área 2. Esses gêneros de cupins 
também foram encontrados por ARRIGONI et al. (1989) num levantamento utilizando-se de outra metodologia, nas regiões de Jaú e Sertãozinho, SP.

Neste trabalho, comprovou-se a eficácia da armadilha Termitrap ${ }^{\circledR}$ estudada por AlmeIDA; Alves (1995) demonstrandoserpossívelutilizá-lanomonitoramento, estudo de bioecologia e controle de H. tenuis.

\section{AGRADECIMENTOS}

Os autores agradecem à FINEP pelo suporte financeiro deste projeto de pesquisa.

\section{REFERÊNCIAS}

AHMAD, M.; AFZAL, M.; SALIHAH, Z. The effects of different relative humidites on survival and moisture loss of workers and soldiers of Heterotermes indicola (Wasman) (Isoptera: Rhinotermitidae) under starvation conditions. Pakistan Journal of Zoology, v.14, n.1, p.65-70, 1982.

ALMEIDA, J.E.M.; ALVES, S.B.. Seleção de armadilhas para a captura de Heterotermes tenuis (Hagen) Anais da Sociedade Entomológica do Brasil, v.24, n.3, p.619-624, 1995.

ALMEIDA, L.C.; PETRI, J.L.; IGLESSIAS, A.F. Flutuação populacional e avaliação de danos por cupins em parcelas tratadas com diferentes inseticidas. Boletim Técnico Copersucar, n.46, p.37-43, 1989.

ARRIGONI, E.B.; ALMEIDA, L.C.; KASTEN JR., P.; PRECETTI, A.A.C.M.. Distribuição de espécies de cupins em cana-de-açúcar, em unidades cooperadas das regiões de Jaú e Sertãozinho-SP. Boletim Técnico Copersucar, n.48, p. 38-47, 1989.

CAMPOS, M.B.S. Aspéctos bioecológico de Heterotermes tenuis (Isoptera: Rhinotermitidae) em cana-de-açúcar. 2004. 132p. Tese (Doutorado em Ecologia e Recursos Naturais) - Universidade Federal de São Carlos, São Carlos, 2004.

FRENCH, J.R.J.; ROBINSON, P.J.; BARTLETT, N.R. A rapid and relative field assessment of termite wood feeding preferences of the subterranean termite Heterotermes ferox (Frogg.) using toilet roll and small wood-block baits. Sociobiology, v.6,n.2, p.135-151, 1981.

GRACE, J.K.; ABDALLAY, A.; FARR, K.P. Eastern subterranean termite (Isoptera: Rhinotermitidae) foraging territories and populations in Toronto. Canadian Entomologist, v.121, p.551-556, 1989.

HAASGMA, K.A.; RUST, M.K. Colony size estimates, foraging trends, and physiological characteristics of the Western Subterranean termite (Isoptera:

Rhinotermitidae). Environmental Entomology, v.24, n.6, p.1520-1528, 1995.

JONES, S.C.; TROSSET, M.W.; NUTTING, W.C. Biotic and abiotic influences on foraging of Heterotermes aureus (Snyder) (Isoptera: Rhinotermitidae).

Environmental Entomology, v.16, n.3, p.791-795, 1987.

KING, E.G.; SPINK, W. T. Development of incipient Formosan Subterranean Termite colonies in the field. Annals of the Entomological Society of America , v.68, n.2, p.355-358, 1975.

NOVARETTI, W.R.T. Controle de cupins em cana-deaçúcar através do emprego de inseticidas de solo. Boletim Técnico Copersucar, n.33, p.39-44, 1985.

THOMPSON, L.R. Bait stake detection of the Formosan termite in south Florida. Florida Entomology, v.68, n.4, p.641-645, 1985.

Recebido em 3/12/08

Aceito em 21/10/09 